\title{
Relação entre a fenologia da espécie Phyla betulifolia (kunth) Greene e elementos climáticos
}

\author{
Relationship between Phyla betulifolia (kunth) Greene phenology and climatic elements \\ Relación entre la fenología Phyla betulifolia (kunth) Greene y los elementos climáticos
}

Recebido: 17/04/2021 | Revisado: 25/04/2021 | Aceito: 30/04/2021 | Publicado: 15/05/2021

\author{
Osmar Alves Lameira \\ ORCID: https://orcid.org/0000-0001-8370-8562 \\ Embrapa Amazônia Oriental, Brasil \\ E-mail: osmar.lameira@embrapa.br \\ Ruanny Karen Vidal Pantoja Portal Moreira \\ ORCID: https://orcid.org/0000-0002-9566-3741 \\ Universidade Federal do Pará, Brasil \\ E-mail: ruanny_vidal@hotmail.com \\ Meiciane Ferreira Campelo \\ ORCID: https://orcid.org/0000-0001-7511-4377 \\ Universidade Federal do Pará, Brasil \\ E-mail: meicianecampelo@gmail.com \\ Simone de Miranda Rodrigues \\ ORCID: https://orcid.org/0000-0001-5717-785X \\ Embrapa Amazônia Oriental, Brasil \\ simone.rodrigues@embrapa.br \\ Allan Cristiam Santos Ramires \\ ORCID: https://orcid.org/0000-0002-0953-7221 \\ Universidade Federal Rural da Amazônia, Brasil \\ E-mail: allanramires15@gmail.com
}

\begin{abstract}
Resumo
A Phyla betulifolia (Kunth) Greene é comumente conhecida como capim-doce merece destaque pelo potencial medicinal. Segundo as comunidades tradicionais da região amazônica, auxilia no controle da diabetes, além de possuir ação calmante. Um dos passos iniciais para o conhecimento das diversas espécies é o estudo de sua biologia e em particular de sua fenologia, pois se refere à parte da botânica que estuda as diferentes fases do crescimento e desenvolvimento das plantas, tanto a vegetativa quanto à reprodutiva demarcando lhes as épocas de ocorrência e as respectivas características. Este trabalho objetivou avaliar a freqüência fenológica reprodutiva de Capim doce relacionando com as condições climáticas de temperatura média do ar e precipitação pluviométrica pretendendo especificar o tempo mais adequado para coletar o material vegetal para uso medicinal e propagação da espécie. Considerando 10 indivíduos de Capim doce, a avaliação de freqüência das fenofases foi realizada matinalmente durante cinco dias por semana registrando-se a ocorrência das fenofases, de 2014 a 2019. O Capim doce apresentou a fase reprodutiva durante todo o período avaliado, ocorre à influência dos elementos climáticos na floração da espécie. A frutificação não sofreu influencia da precipitação pluviométrica e temperatura média do ar. A coleta de material vegetal desta espécie para o uso medicinal deve ser realizada no período de menor número de dias das fenofases e para a propagação pode-se coletar durante todo o ano.
\end{abstract}

Palavras-chave: Fenofases; Precipitação pluviométrica; Temperatura média do ar.

\begin{abstract}
Phyla betulifolia (Kunth) Greene is commonly known as sweet grass and deserves to be highlighted for its medicinal potential. According to traditional communities in the Amazon region, it helps in controlling diabetes, in addition to having a calming action. One of the initial steps for the knowledge of the different species is the study of their biology and in particular of their phenology, since it refers to the part of botany that studies the different stages of growth and development of plants, both vegetative and reproductive, demarcating them the times of occurrence and the respective characteristics. This work aimed to evaluate the reproductive phenological frequency of Capim doce, relating to the climatic conditions of average air temperature and rainfall, aiming to specify the most appropriate time to collect the plant material for medicinal use and propagation of the species. Considering 10 individuals of Capim Doce, the frequency evaluation of the phenophases was carried out in the morning for five days a week, recording the occurrence of the phenophases, from 2014 to 2019. The Capim Doce presented the reproductive phase during the entire evaluated period, occurs under the influence of climatic elements in the flowering of the species. Fruiting was not influenced by rainfall and average air temperature. The collection of plant material of this species for medicinal use must be carried out in the period of the least number of days of the phenophases and for the propagation it can be collected throughout the year.
\end{abstract}


Keywords: Phenophases; Rainfall precipitation; Average air temperature.

\section{Resumen}

Phyla betulifolia (Kunth) Greene se conoce comúnmente como hierba dulce y merece ser destacada por su potencial medicinal. Según las comunidades tradicionales de la región amazónica, ayuda a controlar la diabetes, además de tener una acción calmante. Uno de los pasos iniciales para el conocimiento de las diferentes especies es el estudio de su biología y en particular de su fenología, ya que se refiere a la parte de la botánica que estudia las diferentes etapas de crecimiento y desarrollo de las plantas, tanto vegetativo como reproductivo. demarcándolos los tiempos de ocurrencia y las respectivas características. Este trabajo tuvo como objetivo evaluar la frecuencia fenológica reproductiva de Capim doce en relación con las condiciones climáticas de temperatura media del aire y precipitaciones, con el objetivo de precisar el momento más adecuado para recolectar material vegetal para uso medicinal y propagación de la especie. Considerando 10 individuos de Capim Doce, la evaluación de la frecuencia de las fenofases se realizó por la mañana durante cinco días a la semana, registrando la ocurrencia de las fenofases, de 2014 a 2019. El Capim Doce presentó la fase reproductiva durante todo el período evaluado, ocurre bajo la influencia de elementos climáticos en la floración de la especie. La fructificación no se vio influenciada por las precipitaciones y la temperatura media del aire. La recolección de material vegetal de esta especie para uso medicinal debe realizarse en el período de menor número de días de las fenofases y para la propagación se puede recolectar durante todo el año.

Palabras clave: Fenofases; Precipitación pluvial; Temperatura media del aire.

\section{Introdução}

A espécie Phyla betulifolia (Kunth) Greene é uma erva perene que alterna de 15-60 cm de comprimento (França et al., 2015). Habita em terrenos baixos, extremos de rios e florestas livres, savanas, pastagens, sendo frequentemente achada em solo arenoso ou argiloso (O’Leary \& Mulgura, 2012).

Pertence à família Verbenácea, que abrange cerca de 30 gêneros e 480 espécies (Atkins, 2004). No Brasil ocorre a maior riqueza da família, com 16 gêneros e 290 espécies, sendo 191 endêmicas (Salimena et al., 2019). Entre as espécies temos a Phyla betulifolia (Kunth) Greene, com ocorrências confirmadas nas regiões Norte (Acre, Amazonas, Pará, Rondônia) Nordeste (Bahia, Pernambuco) Centro-Oeste (Goiás, Mato Grosso do Sul) e Sudeste (Minas Gerais).

Comumente conhecida como capim-doce merecendo destaque pelo potencial medicinal. Segundo as comunidades tradicionais da região amazônica, auxilia no controle da diabetes, além de possuir ação calmante (Ribeiro et al.,2017).

Em vista disso, um dos passos iniciais para o conhecimento das diversas espécies é o estudo de sua biologia e em particular de sua fenologia, pois se refere à parte da botânica que estuda as diferentes fases do crescimento e desenvolvimento das plantas, tanto a vegetativa (germinação, emergência, crescimento da parte aérea e das raízes) quanto à reprodutiva (florescimento, frutificação e maturação), demarcando lhes as épocas de ocorrência e as respectivas características (Câmara, 2006).

Para Souza et al., (2014) a importância da fenologia está relacionada ao suporte oferecido aos estudos da biologia reprodutiva, na coleta de frutos e semente e dispersão, analisando, sobretudo a intensidade e freqüência do evento fenológico e o sincronismo das espécies vegetais. Compreendendo o estudo dos eventos periódicos naturais envolvidos no ciclo biológico das plantas, estimando-se os estádios fenológicos, tais como: florescimento, frutificação e desenvolvimento do fruto (Gonçalves, 2018).

O conhecimento da fenologia reprodutiva é fundamental para embasar o estabelecimento de critérios científicos como coleta de material vegetal com potencial de utilização para os mais diversos fins como: frutícolas, silviculturais e medicinais (Guilherme et al.,2011).

Os eventos fenológicos conforme citado por Alberti et al. (2011) e Ferrera (2012) estão diretamente relacionados aos fatores ambientais, pois são estes que comumente influenciam os fenômenos biológicos. Desta forma é de fundamental importância que se conheça os processos envolvidos nas relações entre clima e fenologia para compreender suas conseqüências, sobretudo diante da variabilidade climática no tempo e no espaço (Bergamaschi, 2007). 
Estudos sobre a fenologia do capim doce é essencial para que se possa identificar o tempo mais apropriado para sua coleta visando a sua utilização. Entretanto, as poucas informações encontradas na literatura o que exibe a escassez desses estudos.

$\mathrm{O}$ trabalho teve como objetivo avaliar a frequiência fenológica reprodutiva do Capim doce relacionando com as condições climáticas de temperatura média do ar e precipitação pluviométrica pretendendo indicar a tempo mais apropriado para coleta do material vegetal para uso medicinal e propagação da espécie.

\section{Metodologia}

Os dados fenológicos foram coletados no horto de plantas medicinais da Embrapa Amazônia Oriental, Belém, Pará, localizado a $01^{\circ} 28^{\prime}$ de latitude sul e $48^{\circ} 27^{\prime}$ de longitude oeste de Greenwich, com umidade relativa do ar $84 \%$ e índice pluviométrico de $3.001 \mathrm{~mm}$ (Bastos et al.,2002).

Realizou-se uma pesquisa em campo, de natureza qualitativa como sugere Pereira et al. (2018) nesse tipo de pesquisa as variáveis são de controle mais complexo que nos estudos laboratoriais. Foram considerados 10 indivíduos de Capim doce (Figura 1), a avaliação da ocorrência dos eventos fenológicos foi realizada matinalmente durante cinco dias por semana, através da coleta de dados de ocorrência de floração considerando botões florais, flores e frutificação, avaliando frutos novos e maduros, durante os anos de 2014 a 2019.

Os dados obtidos foram submetidos à análise de variância e teste de médias Scott-Knott ao nível de 5\% de probabilidade, utilizando o programa estatístico SISVAR (Ferreira, 2011). Foram elaborados gráficos expondo as médias de dias de ocorrência dos eventos fenológicos reprodutivos relacionando-os com as médias mensais do índice de precipitação e da temperatura média do ar com o auxílio do programa Excel. Os dados climatológicos do período estudado foram disponibilizados pela estação meteorológica da Embrapa Amazônia Oriental-Belém, Pará.

Figura 1. Espécie capim doce.

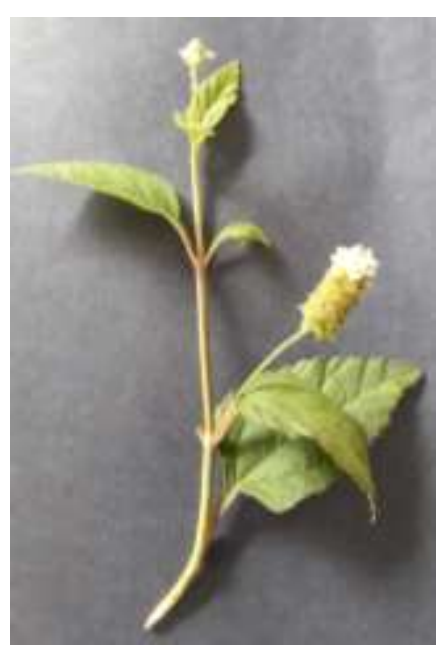

Autor: Autores (2020).

\section{Resultados e Discussão}

Conforme o resultado da análise de variância as médias mensais não diferiram estatisticamente para as variáveis fenológicas reprodutivas, ao nível de significância de $p<0,05$, que apresentaram, respectivamente, as médias gerais de $12,15 \mathrm{e}$ 10,52 dias (Tabela1). 
Tabela1. Análise de variância (ANOVA) para as fenofases reprodutivas do capim doce, índices pluviométricos e temperatura média do ar.

\begin{tabular}{lccc}
\hline Variável & GL & F \\
\hline Floração & & 11 & $0.980 \mathrm{~ns}$ \\
Meses & 12.15 & & \\
Média geral & & & $0.779 \mathrm{~ns}$ \\
Frutificação & & 11 & \\
Meses & 10.52 & & \\
Média geral & & \\
\hline
\end{tabular}

ns-não significativo ao nível de 5\% de probabilidade ( $\mathrm{p}>=0.05$ ). Fonte: Autores (2020).

Ocorreram as fenofases estudadas durante todo o período avaliado. O maior número de dias de floração foi em outubro com média de 18 dias e índice pluviométrico de 113,6 mm, considerado baixo para o período chuvoso amazônico segundo Bastos et al., (2002). Corroborando os resultados do presente estudo, avaliando as fenofases do Capim doce, Portal et al., (2017) obtiveram resultados semelhantes. Segundo Freitas et al., (2015), variações na precipitação pluviométrica e transição seco/chuvoso influenciam a fenofase de floração.

A fenofase frutificação registrou 15 dias de ocorrência em março e outubro meses com distintos índices pluviométricos 498,9 e 113,6 mm nessa ordem, fato que permite o descarte de possíveis relações do evento de frutificação em função da precipitação pluviométrica (Figura 2). Campelo et al., (2018) observaram que a fenofase frutificação na espécie medicinal Solidago microglossa DC. apresentou o pico de atividade em agosto, combinando com baixo índice pluviométrico, para a mesma área de estudo.

Figura 2. Média do número de dias de floração e frutificação do Capim doce e da precipitação pluviométrica.

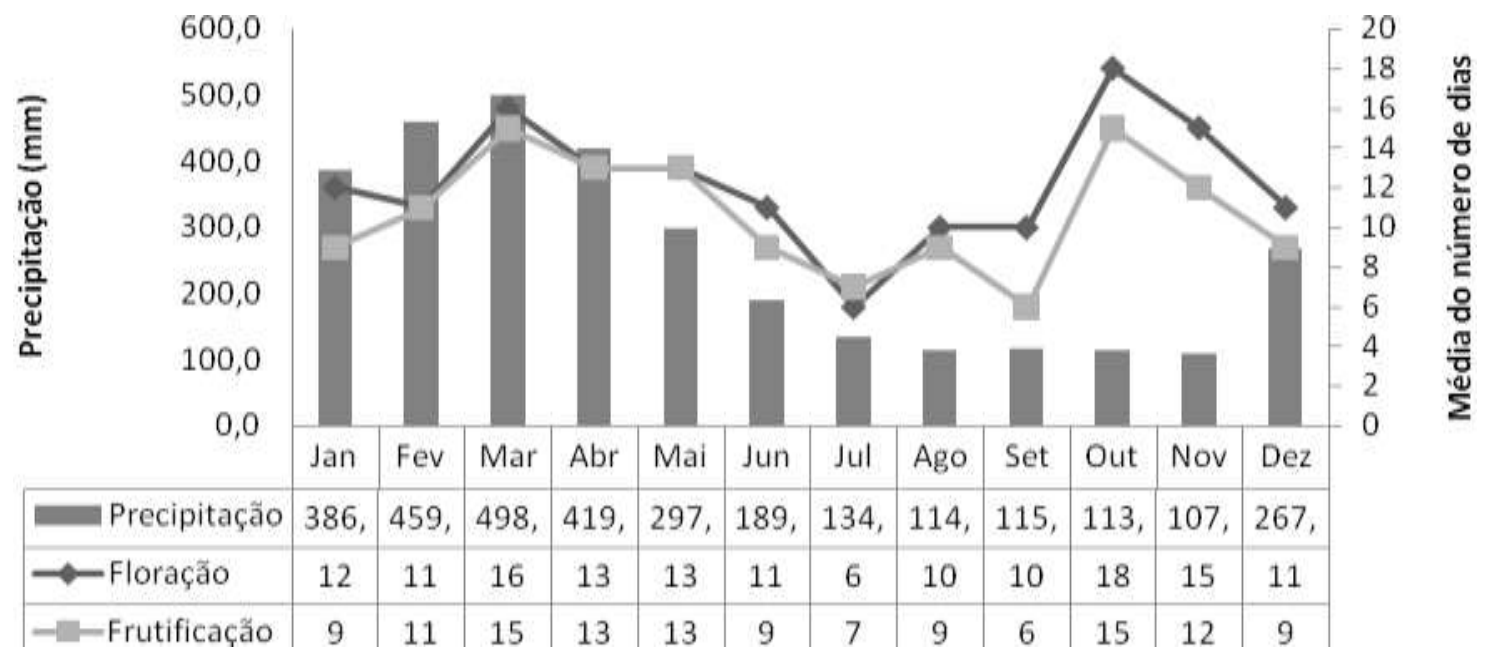

Fonte: Autores (2020).

$\mathrm{O}$ registro das temperaturas médias do ar no período avaliado variou entre 26,3 a $27,9^{\circ} \mathrm{C}$ de fevereiro e novembro respectivamente. Relacionando com os dados dos eventos fenológicos nota-se que a maior média de floração foi registrada em outubro com 18 dias de ocorrência do evento e $27,8^{\circ} \mathrm{C}$ temperatura média do ar, valor próximo à média máxima alcançada no local de estudo. A frutificação teve seu pico em março e outubro ambos com médias de 15 dias com temperaturas de 26,6 e 
27,8 ${ }^{\circ} \mathrm{C}$ essas variações de temperaturas médias observadas são consideradas registros baixos e altos para a região em estudo. Resultados semelhantes foram encontrados por Campelo et al., (2021), em estudo com a espécie Curauá que apresentou o pico de frutificação com maior destaque no mês de outubro, coincidindo com baixos valores de precipitação pluviométrica e elevada temperatura média do ar.

Semelhante ao observado em função da precipitação a floração apresenta certa relação com a temperatura média do ar, entretanto, a frutificação não foi influenciada se considerando a temperatura (Figura 3). De acordo com Hidalgo-Gavez et al., (2018) o elemento climático temperatura exerce influência sobre as alterações fenologicas das espécies vegetais de um determinado local, podendo controlar a resposta fenológica das espécies (Fontana et al., 2018; Richardson et al., 2018). Santana et al., (2020) observaram que o elemento temperatura do ar apresenta influência sobre a fenologia dos acessos de Physalis spp.

Figura 3. Médias mensais de temperatura média para os dias de floração e frutificação do Capim doce.

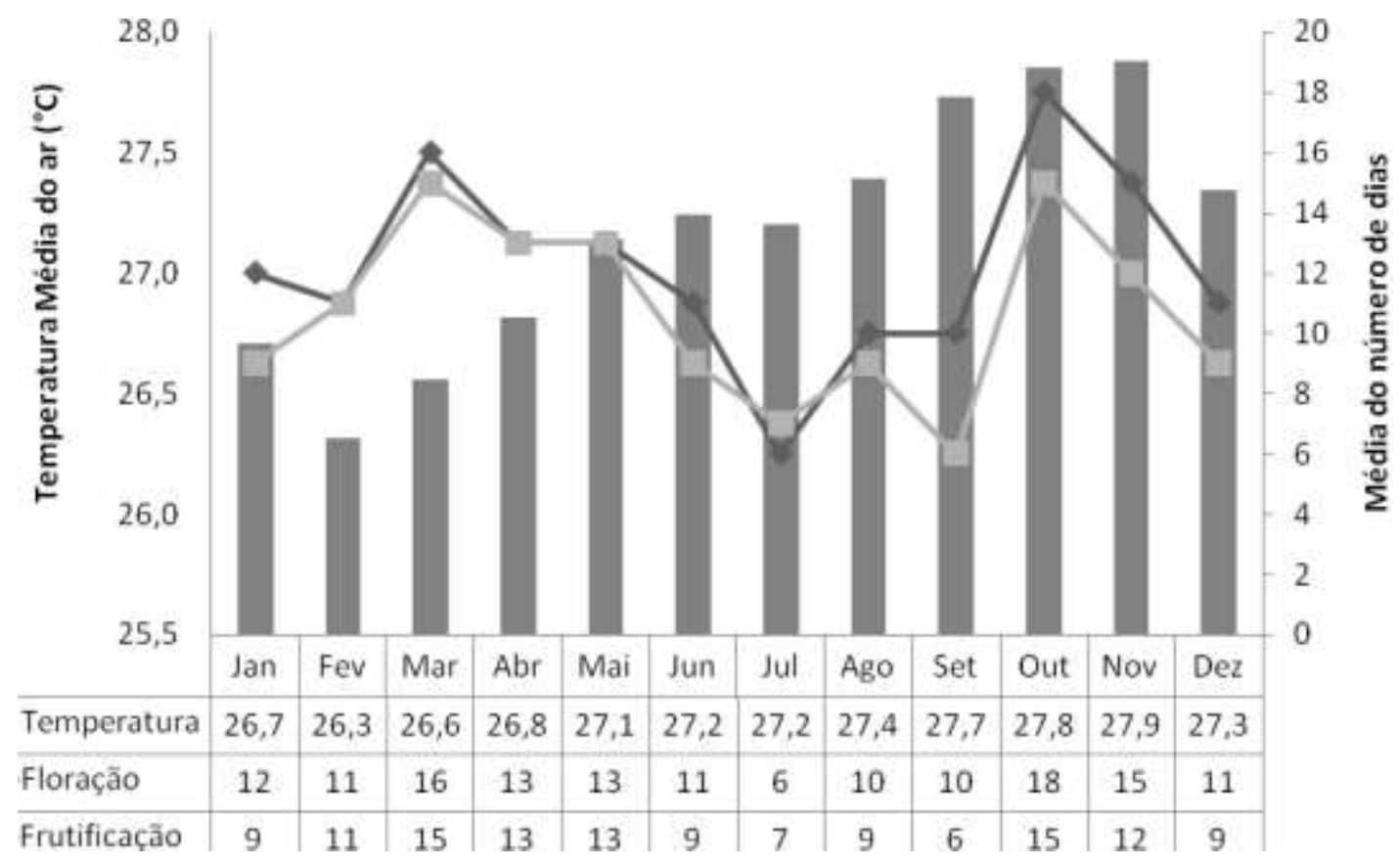

Fonte: Autores (2020).

\section{Conclusão}

A espécie Capim doce apresenta a fase reprodutiva durante todos os meses do ano, sendo que o número de dias mais expressivo de floração ocorre quando há uma queda nos valores registrados de índice de precipitação e alta temperatura média do ar demonstrando a influência destes elementos climáticos na fenologia da espécie, o evento de frutificação não apresentou relação com os elementos climatológicos em estudo. Para coletar material vegetal desta espécie visando o uso medicinal deve ser realizada no período de menor número de dias das fenofases e para a propagação pode-se coletar durante todo o ano.

\section{Referências}

Alberti, L. F., Longhi, S. J, \& Morellato, P. C. (2011). Padrão fenológico de árvores e a relação com o clima em floresta estacional no Sul do Brasil. In Schumacher, M. V. et al. (Orgs.). A floresta estacional subtropical: caracterização e ecologia no rebordo do Planalto Meridional. Editora Pallotti.105-119.

Alongi, D. M. Mangrove forests: resilience, protection from tsunamis, and responses to global climate change. (2008). Estuarine, CoastalandShelf Science, 76(1), 1-13. https://doi.org/10.1016/j.ecss.2007.08.024.

Atkins, S. Verbenaceae. I. N.: \& Kadereit, J. W. (2004). The families and genera of flowering plants.Springer-Verlag, 7, 449-468. 
Bastos, T. X., Pacheco, N. A., Nechet, D., \& Sá, T. D. A. (2002). Aspectos climáticos de Belém nos últimos cem anos. Embrapa Amazônia Oriental-Documentos (INFOTECA-E).

Bergamaschi, H. O clima como fator determinante da fenologia das plantas. In: Rego, C. M.; Negrelle, R. R. B.; Morelatto, L. P. C. (2007). Fenologia: ferramenta para conservação, melhoramento e manejo de recursos vegetais arbóreos.Colombo: Embrapa Florestas. 291-310.

Câmara, G. D. S. Fenologia é ferramenta auxiliar de técnicas de produção. (2006). VisãoAgrícola,5,63-66.

Campelo, M., Lameira, O., Portal, R., Souza, I. N., \& Pacheco, N. (2018). Caracterização fenológica da arnica (Solidago microglossa DC.). Enciclopédia Biosfera, 15(28).

Campelo, M. F., Lameira, O. A, Moreira, R. K. V. P. P., \& Ramires, A. C. S. (2021). Fenologia reprodutiva de Ananas comosus var. erectifolius (L. B. Smith) Coppens \& F. Leal. e sua correlação com a temperatura média do ar e precipitação. Research, Society and Development, 10 (5). http://dx.doi.org/10.33448/rsdv10i5.14621.

Ferreira, D. F. (2011).Sisvar: A computerstatisticalanalysis system. Ciência e Agrotecnologia, 35 (6), 1039-1042. https://doi.org/10.1590/S141370542011000600001

Ferrera, T. S. Fenologia de espécies arbóreas nativas no jardim botânico da Universidade Federal de Santa Maria, Santa Maria - RS. (2012). 104 f. Dissertação (Mestrado em Agrobiologia) -Universidade Federal de Santa Maria.

Fontana, D. C., Caron, B. O., Schmidt, D., Meira, D., Zanatta, T. P., Brezolin, P., \& Werner, C. J. (2018) .Growth of escarole in different seasons of year, RS, Brazil. Semina: Ciências Agrárias, 39 (2), 455-466.

França, F., O'Leary, N., \& Salimena, F. R. G. (2015). Phylain:Lista de Espécies da Flora do Brasil. Jardim Botânico do Rio de Janeiro. <http://floradobrasil.jbrj.gov.br/jabot/floradobrasil/FB21459>.

Freitas, J. da L., Silva, R. B. L., Cruz Júnior, F. de O., Barbosa Filho, M. N. \& Cantuária, P. de C. (2015). Fenologia reprodutiva de cinco espécies arbóreas em ecossistema de terra firme na Amazônia Brasileira.Biota Amazônia,5 (2), 3844. <http://dx.doi.org/10.18561/21795746/biotaamazonia.v5n2p38-44>.

Gonçalves, B. H. L. (2018). Avaliação fenológica e nutricional de três cultivares de abacateiro em clima subtropical do estado de São Paulo. 124 f.Tese (Doutorado em Agronomia)- Faculdade de Ciências Agronômicas da Unesp - Campus de Botucatu.

Guilherme, F.A.G., Salgado, A.A., Costa, E.A., \& Zortéa, M. (2011). Fenologia de Cybistaxantisyphilitica (MART.) MART. (Bignoniaceae) na região urbana de Jataí,Goiás. BioscienceJournal, 27, 138-147.

Hidalgo-Galvez, M. D., García-Mozo, H., Oteros, J., Mestre, A., Botey, R., \& Galán, C. (2018). Phenological behaviour of early spring flowering trees in Spain in response to recent climate changes. Theoretical and Applied Climatology, 132 (1-2), 263-273.

O'Leary, N., Múlgur A, M. E. A. (2012) Taxonomic Revision of the Genus Phyla (Verbenaceae) Annals: of the Missouri Botanical Garden, 98(4), 578-596. http://dx.doi.org/10.3417/2009120.

Pereira, A. S., Shitsuka, D. M., Parreira, F. B. \&Shitsuka, R. (2018). Metodologia dapesquisa científica UFSM. https://repositorio.ufsm.br/bits tream/handle/1/15824/Lic_Computacao_MetodologiaPesquisa-Cientifica.pdf?sequence=1.

Portal, R. K. V. P., Lameira, O. A., Souza, I. N. G., \& Costa, A. D. S.(2017). Aspecto fenológico da espécie Phyla betulifolia (Kunth) Greene.(Verbenaceae). In: Embrapa Amazônia Oriental-Artigo em anais de congresso (ALICE). In: Seminário De Iniciação Científica Da EMBRAPA Amazônia Oriental, 21, Belém, PA. Anais. Belém, PA: Embrapa Amazônia Oriental.

Ribeiro, F. N.S., DE Assis, R. M., Medeiros, A. P. R., Lameira, O. A., Bertolucci, S. K. V., \& Pinto, J. E. B. P. (2017). Acúmulo de biomassa em Phyla betulifolia (Kunth) Greene em função de diferentes doses de esterco de codorna. In: XXVI Congresso da Pós-graduação Congresso de Iniciação Científica da UFLA.

Richardson, A. D., Hufkens, K., Milliman, T., Aubrecht, D. M., Furze, M. E., Seyednasrollah, B., \& Warren, J. M. (2018).Ecosy stem warming extends vegetation activity but heightens vulnerability to cold temperatures. Nature, 560, 368-371.

Salimena, F.R.G. Stachytarpheta. In: Lista de espécies da flora do Brasil. Jardim Botânico do Rio de Janeiro. <http://floradobrasil.jbrj.gov. br/jabot/floradobrasil/FB15189>.

Santana, A. S., Giacobbo, C. L., do Prado, J., Uberti, A., Louis, B., \& Alberto, C. M. (2020). Fenologia e qualidade de frutos de acessos de Physalis spp. Agrarian, 13(47), 1-8.

Souza, D. N. N., Camacho, R. G. V., de Melo, J. I. M., da Rocha, L. N. G., \& Silva, N. F. (2014). Estudo fenológico de espécies arbóreas nativas em uma unidade de conservação de caatinga no Estado do Rio Grande do Norte, Brasil. Biotemas, 27(2), 31-42. 